Review

\title{
Are Microbes Thermodynamically Optimised Self-Reproducing Machines?
}

\author{
Nima P. Saadat ${ }^{1,+}+\mathbb{D}$, Tim Nies ${ }^{1,+(D)}$, Yvan Rousset ${ }^{1,+}(\mathbb{D})$ and Oliver Ebenhöh $1,2, *(\mathbb{D})$ \\ 1 Institute for Quantitative and Theoretical Biology, Heinrich-Heine-Universität Düsseldorf, \\ Universitätsstraße 1, 40225 Düsseldorf, DE \\ 2 Cluster of Excellence on Plant Sciences (CEPLAS), Heinrich-Heine-Universität Düsseldorf, \\ Universitätsstraße 1, 40225 Düsseldorf, DE \\ * Correspondence: oliver.ebenhoeh@hhu.de \\ + These authors contributed equally to this work.
}

\begin{abstract}
To understand microbial growth with mathematical models has a long tradition that dates back to the pioneering work of Jacques Monod in the 1940s. Growth laws are simple mathematical expressions that aim at describing growth rates of microbes as functions of external parameters, in particular nutrient concentrations. These laws are now widely applied to construct, e.g., dynamic ecosystem models. However, to explain the growth laws from underlying (first) p rinciples is extremely challenging. In the second half of the $20^{\text {th }}$ century, numerous experimental approaches aimed at precisely measuring heat production during microbial growth to determine the entropy balance in a growing cell and to quantify the exported entropy. This has led to the development of thermodynamic theories of microbial growth, which have generated fundamental understanding and identified principle limitations of the growth process. Whereas these approaches considered a growing microbe as a black box, modern theories heavily rely on genomic resources to describe and model genome-scale networks to explain microbial growth. Interestingly, however, thermodynamic constraints are often included in modern modelling approaches only in a rather superficial fashion, and it appears that recent modelling approaches and classical theories are disconnected fields. In order to stimulate a closer interaction between these fields, we here review various theoretical approaches that aim at describing microbial growth based on thermodynamic principles. We start with classical black-box models of cellular growth, and continue with genome-scale modelling approaches that include thermodynamics, before we place these models in the context of fundamental considerations based on non-equilibrium statistical mechanics. We conclude by identifying conceptual overlaps between the fields and suggest how the various types of theories and models can be integrated. We outline how concepts from one approach may help to inform or constrain another, and we demonstrate how genome-scale models can be used to infer classical black-box parameters, which are experimentally accessible in growth experiments. Such integration will allow understanding to what extent microbes can be viewed as thermodynamic machines, and how close they operate to theoretical optima.
\end{abstract}

Keywords: Energy; Entropy; Anabolism; Catabolism; Microbial cultures; Biotechnology

\section{Introduction}

Life is certainly one of the greatest wonders on earth. The ability to grow and reproduce distinguishes living systems fundamentally from inanimate objects and engineered devices. However, of course any living organism is also subject to universal physical laws. As such, life is nothing special. No surprise, therefore, that generations of scientists have studied life, and in particular 
microbial growth, using concepts and theories from physics. Whether microbes can be considered as sophisticated biochemical machines is a deeply philosophical and highly relevant question [1]. We do not wish to enter this discourse here, and rather adopt a pragmatic point of view. Certainly, every microbe uses available free energy gradients (either from chemicals in the environment, or - in the case of photosynthetic organisms - from sunlight) to convert chemicals from the environment into a copy of itself. Thus, microbes are not fundamentally different from machines, except that they do not convert free energy gradients into mechanical work that is useful for all kinds of purposes, but into chemical work, which is used to construct a copy of themselves. So why should not concepts, principles, and formulas from thermodynamics, which can so well describe steam engines, be applicable also to microbial growth? In fact, it was exactly this mindset that led generations of scientists to develop excellent thermodynamic theories of microbial growth in the second half of the $20^{\text {th }}$ century. Notably, these researchers had only very limited knowledge of the detailed biochemical processes inside a cell, which are responsible for the macroscopic behaviour. Not surprisingly, the theories consider a microbe as a black box. This view matches well with the available experimental technologies at that time [2,3]. In bioreactors and fermenters, fluxes of matter and energy into and out of the cells could be precisely measured, and, together with observed growth rates, were ideal to challenge the developed theories [4-7].

With the advent of high-throughput technologies, we have now an increasingly detailed picture of the internal processes within a microbe. Especially metabolism, the biochemical networks converting chemicals into biomass, is described with increasing detail. Consequently, new modelling techniques and theories have emerged to describe and investigate genome-scale metabolic networks. Unfortunately, these modern approaches now form a separate research field that appears to be unconnected to existing thermodynamic theories of life. Scientists developing genome-scale network models and corresponding analysis techniques often seem to be unaware of the fundamental thermodynamic theories and thus ignore an invaluable heritage left behind by generations of great minds.

The purpose of this review is to provide a summary of essential developments of theory building, both from the times before high-throughput technologies and after. We aim at developing links and connections between modern modelling approaches and thermodynamic theories of microbial growth, with the hope that these existing and promising research fields can mutually benefit from each other. Genome-scale metabolic network models provide an unprecedented opportunity to peek inside the black boxes. In conjunction with recent developments of non-equilibrium thermodynamics to describe self-replication, we are convinced that an integration of the different approaches to describe microbial growth offers the chance to discover fundamental principles of life.

\section{Historical Overview}

The economy of energy flow in living matter is a fascinating and complex topic that is and was investigated by generations of great scientists. Starting with the pioneering work of Jaques Monod [8-10], this section aims to give a concise overview of the historical developments in the treatment of thermodynamic aspects in models describing microbial growth. By including not only pure theoretical work but also past biotechnological examples, this part illustrates the motivation of the researchers to focus on energetic aspects of microbial cultures, thereby uncovering numerous interesting relationships.

Starting with Monod in the 1940s [9], who defined the biomass yield factor as the amount of biomass formed divided by the amount of limiting nutrient utilized (Y), the quantitative description of the production capabilities in microbial cultures evolved into a whole theory. The introduction of a simple hyperbolic relationship that connects the amount of limiting resources in the environment to growth rates of organisms opened the door for many different modeling approaches. Even today, the Monod growth law together with modern mathematical tools forms the basis for sophisticated theories of microbial growth. Although the work of Jaques Monod is undoubtedly one of the major achievements in the life sciences of the 20th century, it is worth mentioning that others have 
81

\section{proposed growth laws with slightly different functional dependencies of the growth rate on substrate availability [11,12]. As Esener et al. show, all three models are able to realistically describe experimental data of batch cultures [13].}

While the description of batch cultivation could be adequately described by Monod's theory, the growth of microbes in continuous cultures needed a different treatment. In 1956 Herbert et al. presented a formal analysis of continuous cultures, introducing fundamental equations for describing those systems efficiently [14]. In addition to this, Herbert realised in 1958 [13,15] that the yield factor introduced in the 1940s by Monod is not constant but changes with the dilution rate. Both Herbert (1958) and Pirt (1965) tried to explain this behaviour by introducing maintenance terms that describe a growth-independent substrate requirement [15,16]. Although Herbert [15] and Pirt [16] provide different explanations for the maintenance term, Esener et al. have argued that both explanations can be used to essentially obtain the same results [13]. For a detailed discussion of different maintenance parameters and their measurement see [17].

With these theories in hand, the question appeared how essential parameters of microbial systems can be easily and relatively quickly estimated, given the diversity of organisms used in the laboratories or in biotechnology, especially regarding the limited information about the metabolism available at that time. The solution was the introduction of so called black box models that only need input and output information, which can be readily obtained by controlling a bioreactor [2,3,13,18-22]. However, since the applicability of black box models heavily depends on energy and mass balance, a profound thermodynamic background is needed to understand and justify these approaches. Black box models were successfully applied to reveal many interesting relationships between energy, biomass and yield.

As Mayberry et al. state in the 1960s, there was a considerable interest in investigating the proportionality between the yield and the converted energy [23]. One important contribution was made by Mayberry et al. in 1968, who observed that for bacteria growing on a single carbon compound that serves as carbon and energy source, the amount of produced biomass per available electrons (av $e^{-}$) is relatively stable (3.14 $\mathrm{gDW} / \mathrm{av} e^{-}$) for a wide range of organic compounds. By redefining the concept of the "degree of reduction", which was first proposed by Gunsalus and Shuster [24], as "[number] of those electrons in a compound not involved in orbitals with oxygen [...]", and using the above mentioned observation, Mayberry et al. stressed the usefulness of counting available electrons for the analysis of growth data. A significant contribution was to introduce the degree of reduction in the treatment of energetic aspects in biology as a measurement of reducing power of a compound. In 1973 Minkevich and Eroshin [25] derived a formula for calculating the degrees of reduction $(\gamma)$ of the electron donor, the biomass and metabolic products as well as a formula to obtain the dried biomass per oxygen using an overall metabolic reaction. However, great care must be taken when calculating and interpreting $\gamma$, since its derivation is dependent on the nitrogen source. Therefore, Roels later defined a generalized formula for the degree of reduction that can be used for any available nitrogen source [19].

By comparing numerous different organisms, carbon sources and literature, Minkevich and Eroshin concluded that the degree of reduction of biomass $\left(\gamma_{b}\right)$ for many species varies around 4.2 (for comparison, glucose and acetate have a degree of reduction of 4 per carbon, while methanol has 6 , and oxalic acid 1). In addition to this, they used old thermochemical knowledge from the 19th and early 20th century, namely that the molar combustion heats of organic compounds are nearly proportional to the consumed oxygen (known as Thornton's rule [26]) to derive an efficiency parameter of growth $(\eta)$, which specifies the energy fraction (contained in the substrate) transferred to biomass. This efficiency parameter sets a thermodynamic upper bound of the yield. Setting $\eta=1$, one obtains an upper bound of $\mathbf{Y}<\frac{\gamma_{s}}{\gamma_{b}}$, where $\gamma_{b}$ denotes the degree of reduction of the biomass and $\gamma_{s}$ of the electron donor $[18,25]$. Nonetheless, this relationship is only valid if both, the biomass and electron donor, possess the same heat of combustion per available electrons. By studying different chemical species, Minkevich and Eroshin confirmed that the heat of combustion per gram-equivalent of oxygen 
consumed or per available electrons is for most organic substances around $26.94 \mathrm{kcal}$. Similar values have been recalculated by numerous authors (for instance see [27,28]).

Later Erickson together with Minkevich and Eroshin introduced two more efficiency measures: The fraction of the energy in an organic compound transferred to products $\left(\xi_{p}\right)$ and the fraction that is evolved as heat $(\epsilon)$ during the growth process. Combined with the aforementioned energy fraction transferred to biomass $(\eta)$, Erickson et al. developed simple mathematical tests to check for the consistency of various measured parameters, including growth and maintenance requirements [18].

However, as Roels states in his excellent report, those parameters are based on a not entirely correct formulation of the second law of thermodynamics for open systems, because they assume that cellular growth always releases heat to the environment [19]. However, there are examples of microbes importing heat from the environment [2,5]. Roels provides a modified definition of maximum yield on substrate that are consistent with the second law, $\omega_{f}$, consistent with the heat transported to the environment, $\omega_{e}$, and consistent with the oxygen transport, $\omega_{0}$. By showing that the values of $\omega_{f}$ and $\omega_{e}$ most often only deviate by $10 \%$, he basically confirmed that Minkevich's and Eroshin's $\eta$ was a useful parameter, at least under aerobic conditions.

Since $\omega_{f}$ is the maximum value of the yield that is consistent with the laws of thermodynamics, Roels defined the thermodynamic efficiency of a growth process as $\eta_{t h}=Y_{s x} / \omega_{f}$, where $Y_{s x}$ is the yield of biomass (x) given a certain substrate (s). By calculating the values of $\eta_{t h}$ for different organic compounds as energy source, he observed that highly reduced as well as highly oxidized substrates lead to a rather low efficiency. Roels concluded that substrates with a degree of reduction above 4.2 (the degree of reduction of biomass) contain enough energy to allow that all carbon could in principle be converted into biomass. In contrast to this, for compounds with a degree of reduction below 4.2, this is not possible due to energetic reasons.

How to incorporate these results into a mathematical model of bacterial growth is extensively discussed by Esener in his report "Theory and Application of unstructured Models: Kinetic and Energetic Aspects" [13]. Together with his paper published in 1982, in which Esener et al. describe how to include aspects of varying biomass composition under a changing environment in a formal description of bacterial growth [29], his work is a good example of an early attempt to develop a consistent model explaining bacterial growth. These approaches are clearly related to modern approaches to explain bacterial growth laws (see [30,31]). For a very well written discussion comparing most of the efficiency measures discussed above (and much more), see [22,32].

In combination, these investigations and concepts of thermodynamic efficiency in bacterial growth provided the basis for further developments, including methods to estimate the energy and entropy of formation for biomass, which represents a parameter of fundamental importance for energetic calculations concerning life $[27,28,33,34]$. Particularly valuable were Battley's contributions for estimating the Gibbs free energy of formation of biomass, $\Delta_{f} G_{b}\left(-65.10 \mathrm{~kJ} / \mathrm{C}-\mathrm{mol}, \gamma_{b}=4.998, \mathrm{~N}_{2}\right.$ as nitrogen source), and the enthalpy of formation for biomass, $\Delta_{f} H_{b}$. By using the well known relation of entropy, enthalpy and Gibbs free energy, $\Delta G=\Delta H-T \Delta S$, he even attempted to estimate the entropy of formation of biomass, but concluded that this method is too prone for errors, because it highly depends on the quality of the approximation of enthalpy and Gibbs free energy [28].

\section{Recent applications of Thermodynamics in microbial growth}

The introduction of novel experimental techniques, often referred to as high-throughput 'omics' technologies, around the beginning of the 21st century, facilitated collecting extensive datasets and information about important molecular components of cells, including metabolite, RNA and protein levels. Falling cost and improved efficiency of genome sequencing gave rise to an ever growing number of sequenced organisms [35]. The availability of omics data also resulted in comprehensive biochemical databases that collect information about enzymatic reactions and their compounds [36, 37]. The construction of genome-scale metabolic models [38] is still a widely used approach to investigate microbial growth rates, using flux balance analysis (FBA) as a method to predict metabolic 
fluxes in optimised growth scenarios [39]. Well curated genome-scale metabolic models can provide valuable information about flux distributions of cells growing on different media. In combination with experimental measurements, this approach has successfully been applied to investigate uptake rates of carbon sources and byproduct secretion rates for several microbial organisms [40-43]. However, there are a number of phenomena associated with microbial growth which cannot be explained by these type of models. For example, they do not explain the observation of fermentative metabolism and only partial oxidation of organic substrates in aerobic conditions and high substrate availability (known as the Crabtree effect in yeast or the Warburg effect in cancer cells) [44]. In general, flux balance analysis is very limited when investigating fundamental principles of microbial growth.

Thermodynamic approaches are typically used in genome-scale metabolic models to avoid infeasible flux distributions in the solution space. Thermodynamics gives information about the correct direction of biochemical reactions [45]. Essentially any enzymatic reaction can be reversible, and the Gibbs free energy of reaction $\left(\Delta_{R} G\right)$ defines the direction in which a reaction proceeds (always in the direction of negative $\Delta_{R} G$ ). The Gibbs free energy of reaction depends on the metabolite concentrations by

$$
\Delta_{R} G=\sum_{i} n_{i} \Delta_{f} G_{i}^{0}+R T \sum_{i} n_{i} \ln c_{i}
$$

where $n_{i}$ is the stoichiometric coefficient of metabolite $i, c_{i}$ its concentration, and $\Delta_{f} G_{i}^{0}$ the respective standard Gibbs energy of formation. However, flux balance analysis does not incorporate metabolite concentrations. Therefore, often physiological substrate concentration ranges are assumed [46], and in combination with - often computationally derived $-\Delta_{f} G^{0}$ values [47], limitations on reaction directionality can be calculated. This approach, if applied correctly, guarantees that only thermodynamically feasible pathways [48] are found in the solution space. With incorrect assignment of reaction directions, infeasible pathways can arise in genome-scale metabolic models and result e.g. in pathways that produce ATP without external driving forces.

In recent years, resource allocation models and models including molecular crowding have been developed to further investigate microbial growth limitations and overflow metabolism [49-51]. Whereas flux balance analysis neglects specific protein costs and benefits, resource allocation models are focusing on incorporating costs for enzymes, ribosomes, available space in cells and other physiological aspects. In fact, understanding microbial growth as an economic process, in which limited resources (such as macromolecules or space) have to be efficiently allocated, dates back to the pioneering work of Schaechter et al. [52,53] in the 1950s. Recently, comprehensive resource allocation models were proposed, which allow calculating and thus further understanding balances and trade-offs during microbial growth [49]. Molenaar et al. argue that it is beneficial to replace a simple biomass flux objective with a more complex objective of self replication, because even the basic self-replicator model introduced in their work exhibits a high degree of complex behaviours [44]. Noor et al. argue that Flux-force relationships show that the logarithm of the ratio of forward and backward fluxes of a reaction is proportional to the Gibbs energy dissipation of the reaction [54], and therefore conclude that reactions with Gibbs energy dissipation close to zero require increased amounts of enzyme to facilitate a given net flux. In most resource allocation models, these thermodynamic contributions are not included. Extensive incorporation of thermodynamic principles has been applied by Niebel et al. [55] to analyze growth of yeast and E. coli, including thermodynamic constraints. In their work, it was suggested, based on experimental data, that an upper limit of the Gibbs energy dissipation rate exists. This assumption allows the formulation of an equation for the Gibbs energy balance. This balance constrains the sum of all Gibbs energy dissipation rates of all internal reactions to be equal to the exchange of Gibbs energy with the environment. The resulting models were used to predict oxygen uptake as well as byproduct and biomass production rates for different substrate uptake rates. Further, some intracellular fluxes were predicted and then measured with ${ }^{13} \mathrm{C}-\mathrm{MFA}$. In their work it was observed that with increasing substrate uptake rates, the model shifted flux distributions from pathways with high dissipation rates, such as respiration, to pathways with lower 
dissipation rates, such as fermentation. Thus they could provide a putative explanation, based on thermodynamic principles, for overflow metabolism phenomena, such as the Crabtree and Warburg effects. In contrast to standard flux balance analysis of genome-scale models, the model presented in their work also incorporates metabolite concentrations. This approach, while certainly increasing thermodynamic rigour and improving the predictive capabilities, comes at the cost of increased computational complexity. To find flux distributions that maximize growth, the authors used a mixed-integer non-linear program.

\section{Thermodynamic approaches to self replication}

In the 1990s, the development of the so-called fluctuation theorems [56-59] constituted a major advance in non-equilibrium statistical physics. Basically, they represent a generalisation of the second law of thermodynamics. They link the probability to observe an entropy increase $\sigma$ during a time $\tau$ to the probability to observe an entropy decrease by the same amount in the same time. This class of theorems can be generally expressed by

$$
\frac{\mathcal{P}(+\sigma)}{\mathcal{P}(-\sigma)}=\exp (\sigma \tau)
$$

where $\mathcal{P}(+\sigma)$ and $\mathcal{P}(-\sigma)$ denote the probabilities to observe an entropy increase or decrease by $\sigma$ during time $\tau$, respectively. More recently, J. L. England [60] has proposed to apply this approach to self-replicating systems. Obviously, replication is a highly irreversible biological process and can be described in the language of statistical physics as a system that goes from a macrostate I (a single cell and the substrates in the surrounding medium), to a macrostate II (two daughter cells and the substrates), where each macrostate corresponds to an extremely high number of microstates. England's reasoning starts from the fact that particles obey classical mechanics at the microscopic scale, and therefore follow a reversible dynamics. This allows quantifying the reversibility of a microscopic transition by the associated change in entropy. Applying these microscopic considerations to the macroscopic scale, the author obtains a generalization of the second law of thermodynamics for macroscopic irreversible biological processes. While the classical second law of thermodynamics states that the increase of entropy of a closed system is always positive and obeys the inequality

$$
\Delta Q_{\text {ex }}+T \Delta S_{\text {int }} \geq 0,
$$

where $\Delta Q_{\text {ex }}$ is the amount of heat exchanged with the environment and $\Delta S_{\text {int }}$ the internal entropy increase of the system. England's derivation adds a new term to this relation,

$$
\frac{\Delta Q_{\mathrm{ex}}}{T}+\Delta S_{\mathrm{int}}+\ln \frac{\pi(\mathbf{I I} \rightarrow \mathbf{I})}{\pi(\mathbf{I} \rightarrow \mathbf{I I})} \geq 0,
$$

where $\pi(\mathbf{I} \rightarrow \mathbf{I I})$ (respectively $\pi(\mathbf{I I} \rightarrow \mathbf{I})$ ) stands for the probability that the system evolves from macrostate I to macrostate II (respectively from II to I). When a macroscopic transition is irreversible $(\pi(\mathbf{I I} \rightarrow \mathbf{I}) \ll \pi(\mathbf{I} \rightarrow \mathbf{I I}))$, the logarithm becomes negative, increasing the lower bounds for heat dissipation and entropy increase.

Equation (4) allows to have a closer look at self-replication. England applies this relation to a population of exponentially growing cells. He denotes the growth rate as $g$ and the reverse rate (highly unlikely to happen) as $\delta$. It allows to express $\pi(\mathbf{I} \rightarrow \mathbf{I I})$ as $g d t$ and $\pi(\mathbf{I I} \rightarrow \mathbf{I})$ as $\delta d t$. Hence, equation (4) becomes

$$
\frac{\Delta q}{T}+\Delta s_{\text {int }} \geq \ln (g / \delta),
$$


where $\Delta q$ and $\Delta s_{\text {int }}$ are the respective intensive quantities. From this relation, one can see that the maximum duplication rate $g_{\max }$ is obtained when the right and left terms are equal. Therefore,

$$
g_{\max }-\delta=\delta\left(e^{\Delta q / T+\Delta s_{\text {int }}}-1\right) .
$$

Because the net growth rate is $(g-\delta)$, this last relation is one of particular interest. The right hand term shows the dependency on three quantities. The maximum growth rate will increase with heat dissipation, $\Delta q$, internal entropy change, $\Delta s_{\text {int }}$, and the rate at which the reverse process would occur, $\delta$. The author emphasizes an interesting property: for identical entropy changes and decay rates, a replicator that dissipates more heat compared to another will have a higher maximum growth rate. A second particularly interesting aspect is the dependency on $\delta$ and $\Delta s_{\text {int }}$. Low degree of organization and low stability make a replicator more competitive.

Application of equation (4) to any self-replicator needs an estimation of the rates $g$ and $\delta$. England considers two examples, a self-replicating RNA and a replicating E. coli cell. For the former, RNA replication is considered to consist of a single ligation, which allows the author to estimate the decay rate $\delta$ from the half-life time of a phosphodiester bond and a duplication rate $g$ from the RNA doubling time. Hence, he found a minimum limit of heat dissipation of $7 \mathrm{kcal} \cdot \mathrm{mol}^{-1}$ for an RNA self-replicator, while the actual heat dissipation for the ligation reaction is measured experimentally as $10 \mathrm{kcal} \cdot \mathrm{mol}^{-1}$. England argues that for the same reaction occurring for DNA, one finds a lower limit for heat dissipation of $16 \mathrm{kcal} \cdot \mathrm{mol}^{-1}$, because of a considerably longer half-life of DNA compared to RNA. Therefore, such a ligation for DNA is thermodynamically not possible.

The same approach is applied to bacterial cell division. England considers a system with a single bacterium at constant temperature $T$ in a rich medium. The system is initially in macrostate $\mathbf{I}$ (a single bacterium and the substrate), and will evolve through cell division to macrostate II (two bacteria and the substrate). Application of equation (4) to this system provides a lower limit on heat dissipation for cell replication. This amount is six times lower than what was experimentally observed for E. coli, which is, according to England, surprisingly close to the thermodynamic limit.

In a very clearly described theoretical framework, Piñero et al. [61] have extended this approach to different types of self-replicating systems. They found specific thermodynamic constraints for each replicator type.

\section{Combining black box techniques with modern genome-scale approaches}

In this section, we attempt to provide examples how to integrate old black box model techniques with modern genome-scale modelling approaches. To integrate thermodynamics into metabolic networks is currently one of the most fascinating and difficult challenges in the field of metabolic pathway analysis. However, methods and concepts developed for black box models are rarely applied in modern theoretical research. Highly relevant quantities are the energies of reaction $\left(\Delta_{R} G\right)$. To determine these requires knowledge of the energies of formation $\left(\Delta_{f} G\right)$ of the participating compounds. In the context of a back box model, only overall reactions are considered, in which nutrients are converted into biomass and byproducts. Therefore, calculating the corresponding energies of reaction depends on the energy of formation of the biomass $\left(\Delta_{f} G_{b}\right)$. This quantity is directly accessible using, for instance, a technique described by Battley in 1993 [28] (see historical overview in Section 2).

To approximate the value of $\Delta_{f} G_{b}$, Battley considers a hypothetical combustion reaction of the biomass into fully oxidised components. For an experimentally determined elemental composition of the biomass of $\mathrm{CH}_{1.595} \mathrm{O}_{0.374} \mathrm{~N}_{0.263} \mathrm{P}_{0.023} \mathrm{~S}_{0.006}$ this reads

$$
\begin{gathered}
\mathrm{CH}_{1.595} \mathrm{O}_{0.374} \mathrm{~N}_{0.263} \mathrm{P}_{0.023} \mathrm{~S}_{0.006}+ \\
1.251 \mathrm{O}_{2}+0.012 \mathrm{KOH} \longrightarrow \mathrm{CO}_{2}+0.131 \mathrm{~N}_{2}+0.006 \mathrm{P}_{4} \mathrm{O}_{10}+ \\
0.006 \mathrm{~K}_{2} \mathrm{SO}_{4}+0.803 \mathrm{H}_{2} \mathrm{O}
\end{gathered}
$$


Employing now the formula to calculate the degree of reduction when $\mathrm{N}_{2}$ is assumed to be the nitrogen source,

$$
\gamma=4 n_{C}+n_{H}-2 n_{O}-0 n_{N}+5 n_{P}+6 n_{S}-n_{e^{-}},
$$

in combination with the relation between the energy of combustion for an organic compound and its degree of reduction (Battley assumes $-107.90 \mathrm{~kJ} / \mathrm{av} e^{-}$), he was able to estimate the energy of formation for biomass as $-65.10 \mathrm{~kJ} / \mathrm{C}$-mol (not including ions). The $n_{X}$ in equation (7) denotes the number of atoms of type $X$ or charge $\left(n_{e^{-}}\right)$, respectively. The energies of formation necessary for obtaining this value are listed in table 1.

Because all necessary information for repeating this calculation are contained in curated genome-scale models, it is rather straight-forward to transfer this old technique to modern modelling approaches. To demonstrate this, we determine the $\Delta_{f} G_{b}$-values for all genome-scale models contained in the BIGG database [62], using the specified biomass reactions. Figure 1 shows the distribution of energies of formation as function of the degree of reduction.

Table 1. Standard free energy of formation for various organic compounds of interest necessary for estimating the energy of formation for biomass (see [28])

\begin{tabular}{cccc}
\hline Substance & \multicolumn{2}{c}{ Formula } & $\Delta_{f} G_{i}^{0}[\mathbf{k J} / \mathbf{m o l}]$ \\
\hline Oxygen & $\mathrm{O}_{2}$ & $(\mathrm{~g})$ & 0 \\
Potassium hydroxide & $\mathrm{KOH}$ & (c) & -379.11 \\
Carbon dioxide & $\mathrm{CO}_{2}$ & (g) & -394.36 \\
Nitrogen & $\mathrm{N}_{2}$ & (g) & 0 \\
Phosphorous decoxide & $\mathrm{P}_{4} \mathrm{O}_{10}$ & (c) & -2697.84 \\
Potassium sulfate & $\mathrm{K}_{2} \mathrm{SO}_{4}$ & (c) & -1321.43 \\
Water & $\mathrm{H}_{2} \mathrm{O}$ & (lq) & -237.18 \\
\hline
\end{tabular}

A comparison with the reported values $\left(\Delta_{f} G_{b}=-65.10 \mathrm{~kJ} / \mathrm{C}-\mathrm{mol}\right.$ and $\gamma=4.998$ - see historical overview in Section 2) reveals that for most models energies of formation and degrees of reduction of the biomass are in agreement with former theoretical approximations. However, there are some values that deviate drastically from the mean (compare, for instance, upper right corner in figure 1). Possibly, the observed variation results from different biomass compositions that were assumed for the specific models and their particular research questions. However, this kind of calculation offers the opportunity to scrutinise the plausibility of model assumptions, in particular referring to the biomass functions. For example, an energy of formation of $+200 \mathrm{~kJ} / \mathrm{C}-\mathrm{mol}$ seems highly unlikely. 


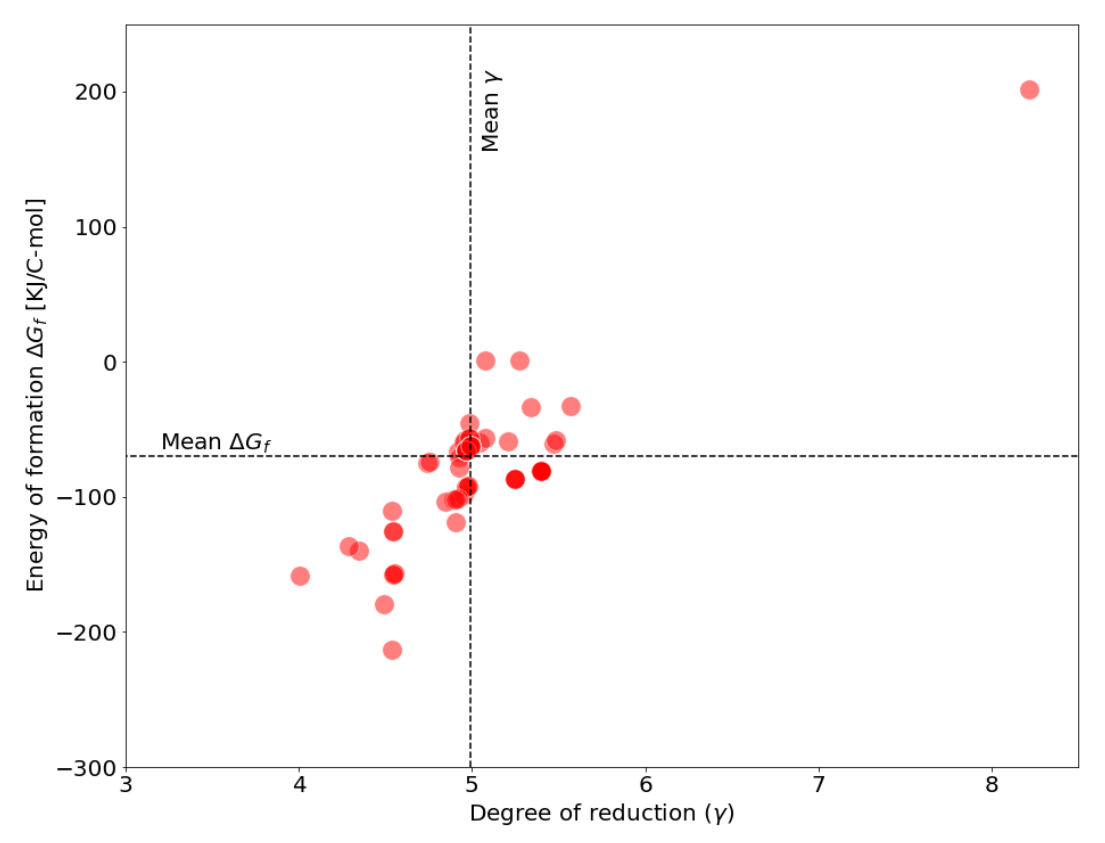

Figure 1. Energy of formation $\Delta_{f} G_{b}$ for biomass as encoded in genome-scale models of the BIGG database. Each point represents a biomass composition as described in the models. 85 models of the BIGG database containing 165 biomass functions were analysed. Mean $\Delta_{f} G_{b}=-70.079 \mathrm{~kJ} / \mathrm{C}-\mathrm{mol}$, Mean $\gamma=4.996$.

Another possibility for a straight-forward approach to combine thermodynamic concepts from black box models with genome-scale models is a separate analysis of anabolism and catabolism. In order to calculate properties of anabolism, such as those predicted by Battley in 1993 [28], from genome-scale models, we pursue the following approach: Genome-scale models from the BIGG database were modified in two steps. First, all reactions that can produce ATP are disabled by introducing a dummy compound representing "unusable" ATP. Second, two strictly coupled reactions are introduced that import ATP into the cytosol, and export ADP and orthophosphate with the same rate. The strict coupling of import and export ensures that only energy but no matter is introduced into the system. Thus, the modified model is unable to produce ATP from any carbon source and instead must use the imported ATP as energy source. Therefore, this modification separates anabolism from catabolism by simulating an external "ATP battery" providing the organism with external chemical energy, replacing the usual catabolic pathways.

These modified models were used to simulate anabolism separate from catabolism. In particular, we calculated the minimum amount of ATP required to incorporate one carbon atom from the nutrients into biomass and the minimum number of $\mathrm{CO}_{2}$ molecules that are released in this process. For this, the biomass production rate was fixed to the value obtained from the original model (in all cases the objective was maximisation of the biomass production rate) and subsequently minimizing all carbohydrate import fluxes. The results are shown in Figure 2. Every point represents one model from the BIGG database. The x-axis displays the ratio of carbon dioxide produced by anabolism only to that produced by the original metabolism, in which anabolism and catabolism are coupled. The $\mathrm{y}$-axis shows how many moles ATP are minimally required to incorporate one mole carbon atoms into the biomass. The large number of data points sharing the same anabolism versus metabolism ratio of released $\mathrm{CO}_{2}$ can be explained by the fact that a large proportion of models available in the BIGG database are for E. coli, and use the same biomass definition. For all E. coli models except the "core 
model", the required ATP per biomass carbon is between 2 and 3.5. Interestingly, the anabolism versus metabolism ratio of released carbon dioxide for the $E$. coli models is very close to the ratio predicted by Battley [28] (indicated by the dotted black line).

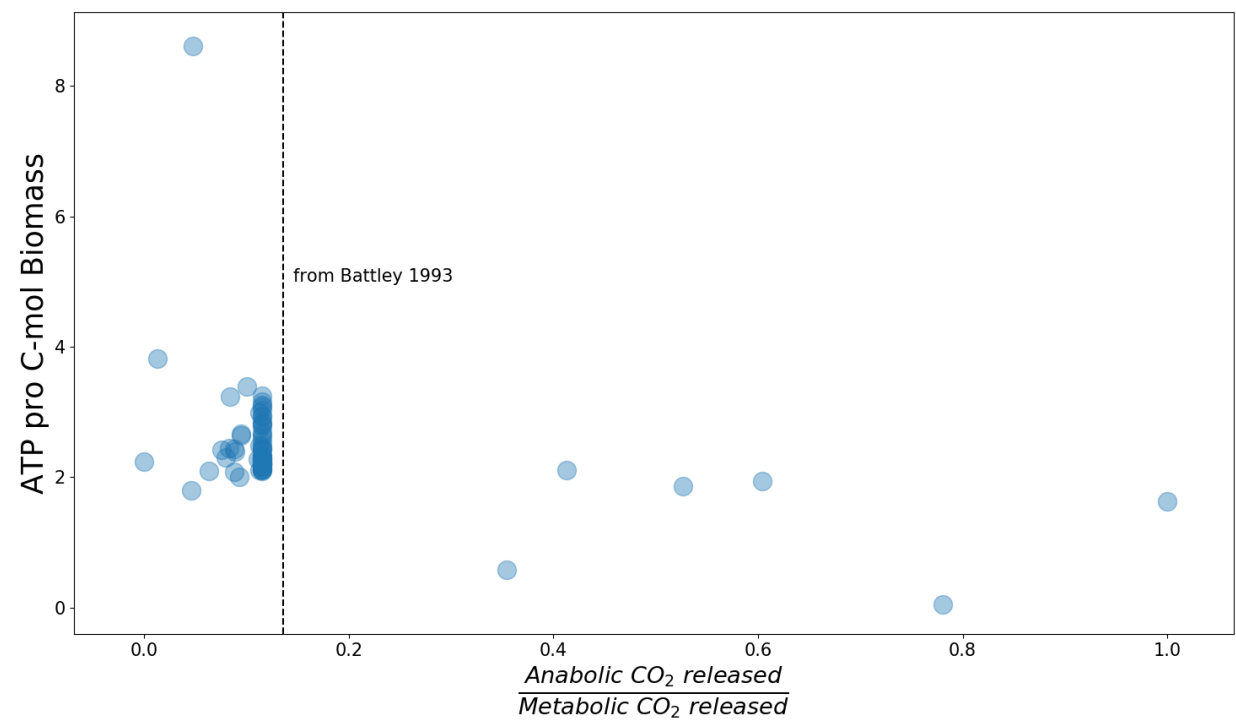

Figure 2. Anabolic properties of genome-scale models of the BIGG database. The y-axis indicates the minimum required amount of ATP per biomass carbon. The x-axis displays the ratio of carbon dioxide released by anabolism to carbon dioxide released by overall metabolism (including anabolism and catabolism).

\section{Outlook \& Conclusion}

To increase fundamental understanding of microbial self-replication and its limitations, thermodynamic considerations and calculations can give valuable insights and expand the current knowledge about microbial growth. When attempting to answer the question whether prokaryotic organisms are thermodynamically optimised self-replicating machines, one is immediately confronted with a number of fundamental problems. There does not seem to be an agreement what exactly "thermodynamic optimality" means. The "black box" models, discussed in section 2, that arose in the 20th century focussed on estimations of microbial growth efficiency parameters by only considering the exchange of matter and energy between cells and their environment. The parameters for aerobic microbial growth efficiency, proposed by Minkevich, Eroshin and Erickson [18,25], incorporate the degree of reduction of the biomass, substrates and electron donors to estimate a thermodynamic upper bound for the yield. This efficiency of biomass yield can be considered an important interpretation of thermodynamic optimality.

The upsurge of high-throughput data in the early 21st century gave rise to genome-scale metabolic models, opening the "black boxes" of microbial metabolism. These models provide excellent tools to investigate metabolic flux distributions. Some genome-scale metabolic models consider thermodynamics to constrain intracellular fluxes, but rarely to find constraints for self-replication and growth itself. The work of Niebel et al. [55], mentioned in section 3, is one of the most comprehensive approaches how to employ thermodynamics for the investigation of microbial growth using genome-scale metabolic models. Based on experimental data, the authors proposed an upper limit of Gibbs free energy dissipation during growth. They supported their hypothesis by implementing additional thermodynamic constraints reflecting this upper limit of Gibbs free energy dissipation. A flux distribution optimising growth, which obeys this upper limit can also be considered as an interpretation of "thermodynamic optimality". A redistribution of fluxes to fermentative pathways due to constraints by the upper limit of Gibbs energy dissipation described by Niebel et al. [55] only occurs at high glucose uptake rates in E. coli and S. cerevisiae. Such conditions 
of excess nutrient availability are rarely found in nature and therefore an evolution towards such thermodynamic optimality is questionable. However, a hypothetical environment of fluctuating periods of excess nutrient availability could provide a fitness increase for microbial organisms adapted to flux redistribution obeying the upper limit of Gibbs energy dissipation.

A promising complementary approach attempts to describe microbial self-replication by first principles from physics. In stark contrast to genome-scale models, which heavily depend on high-throughput data and computational power, first principle concepts require only a minimum amount of data. The work by England, mentioned in section 4, aims at understanding microbial growth from a thermodynamic perspective. By applying fluctuation theorems to the non-equilibrium process of self-replication, a generalization of the second law of thermodynamics for irreversible transitions between two macroscopic states has been derived. This allowed calculating a lower bound for the produced heat during self-replication as a function of the internal entropy, growth and decaying rates. A consequence of these calculations is that a self-replicating microbe that dissipates heat with a rate close to the thermodynamic minimum is optimal in the sense that energy loss is minimised. However, the maximal rate of self-replication increases with increased heat dissipation. The finding that the heat dissipation of E. coli is not far from the calculated minimum needed for self-replication hints at evolution towards thermodynamic efficiency. However, the calculations imply that replication rates are increased with higher internal entropy and an increased rate of spontaneous self-decay. Both properties are not commonly found in microbial organisms. These properties may be beneficial to increase growth rate from a thermodynamic perspective, but are probably disadvantageous regarding other evolutionary pressures.

Evidently, there is no simple and unique answer to the question whether microbes are thermodynamically optimised self-replicating machines. Although all three concepts described here are concerned with the same phenomenon, each represents a different perception and viewpoint on thermodynamic optimality of microbial growth. In our opinion these three concepts, as different as they may be, host an enormous potential to complement each other into an extended understanding of thermodynamic limitations and optimality of microbial growth.

The minimum amount of heat dissipation, and the upper limit of Gibbs free energy dissipation define fundamental thermodynamic limitations of microbial growth. The lower bound is a consequence of the extended second law of thermodynamics: It is impossible to replicate with less dissipated heat. The upper bound is an empiric observation which so far has not experienced a theoretical explanation. It implies that there is a principle upper limit for microbial growth rates. In addition, black box models allow calculating upper bounds of the yield based on physical-chemical properties of substrate and biomass, most importantly the degree of reduction. It is not yet clear how these three limitations can be considered simultaneously, whether they are in agreement, and how combining them might result in a more confined thermodynamic space. An interesting observation was made by von Stockar [5], who reviewed thermodynamic data on microbial growth. This review includes examples of microbes that import heat from their environment, and compensate this by exporting chemical entropy [2]. Other microbes can do the opposite and even reduce the chemical entropy of their environment, at the expense of increased heat dissipation [63].

After pointing out that by combining the different interpretations of thermodynamics of microbial growth their potential could be drastically enhanced, we suggest that attempts to merge different thermodynamic theories should be further intensified. One example of such a combined approach is the analysis of genome-scale models using constraints and concepts from black box models of microbial metabolism. Section 5 shows that in a relatively simple fashion, the combination of genome-scale models and black box models provides promising strategies to further understand thermodynamics of microbial growth and metabolism. However, to incorporate black box model concepts, metabolic networks need to guarantee to obey fundamental conservation laws. For example, calculating the upper thermodynamic limit of the yield $\left(\mathbf{Y}<\frac{\gamma_{s}}{\gamma_{b}}\right)$, requires the determination of the degrees of reduction for the biomass and all substrates. This heavily depends on a fully mass- and charge-balanced 
metabolic network. During our analysis, we noticed that only few models can actually ensure this. This is understandable if one considers that most models were constructed for a completely different purpose. Finding flux distributions that optimise the incorporation of carbon into biomass also work without a perfectly charge-balanced network, and even an incorrect mass balance, which only concerns hydrogen atoms, will not affect the results. Nonetheless, in order to be truly reusable, in particular for thermodynamic calculations, any genome-scale model that is published should adhere to these fundamental chemical principles. We hope that this review encourages further activities to integrate different thermodynamic concepts and motivates the introduction of stricter standards to ensure reusability of genome-scale metabolic models.

Supplementary Materials: All code for analysing published genome-scale metabolic networks in Section 5 is available at https ://gitlab.com/qtb-hhu/thermodynamics-in-genome-scale-models.

Author Contributions: conceptualization, O.E.; validation, N.S., T.N., Y.R. and O.E.; formal analysis, N.S., T.N. and Y.R.; investigation, N.S., T.N. and Y.R.; writing-original draft preparation, N.S., T.N. and Y.R.; writing-review and editing, N.S., T.N., Y.R. and O.E.; funding acquisition, O.E.

Funding: This work was funded by the Deutsche Forschungsgemeinschaft (DFG, German Research Foundation) under Germany's Excellence Strategy - EXC-2048/1 - project ID 390686111 (OE) and project ID 391465903/GRK 2466 (TN), and by H2020 Marie Skłodowska-Curie Actions - grant number: 812616 (YR).

Conflicts of Interest: The authors declare no conflict of interest. The funders had no role in the design of the study; in the collection, analyses, or interpretation of data; in the writing of the manuscript, or in the decision to publish the results.

\section{Abbreviations}

The following abbreviations are used in this manuscript:

Y Biomass yield

$\gamma \quad$ Degree of reduction

$\Delta_{R} G \quad$ Gibbs free energy of reaction

$\Delta_{f} G_{b} \quad$ Gibbs free energy of formation for biomass

$\Delta_{f} G_{i}^{0} \quad$ Standard Gibbs free energy of formation for a metabolite $i$

$\sigma \quad$ Entropy production

${ }_{18} \tau$ Time

$T \quad$ Temperature in Joule, $k_{B}$ set to 1

$\mathcal{P}( \pm \sigma) \quad$ Probability to observe an entropy production of $\pm \sigma$

$\pi(\mathbf{I I} \rightarrow \mathbf{I}) \quad$ Probability to observe a transition from macrostate II to I

$\pi(\mathbf{I} \rightarrow \mathbf{I I}) \quad$ Probability to observe a transition from macrostate I to II

$g \quad$ Duplication rate

$\delta \quad$ Decaying rate

\section{References}

1. Nicholson, D.J. Is the cell really a machine? Journal of Theoretical Biology 2019, 477, 108-126. doi:10.1016/j.jtbi.2019.06.002.

2. Marison, I.W.; von Stockar, U. The application of a novel heat flux calorimeter for studying growth of Escherichia coli W in aerobic batch culture. Biotechnology and Bioengineering 1986, 28, 1780-1793. doi:10.1002/bit.260281205.

3. Marison, I.; Liu, J.S.; Ampuero, S.; Von Stockar, U.; Schenker, B. Biological reaction calorimetry: development of high sensitivity bio-calorimeters. Thermochimica Acta 1998, 309, 157-173.

4. von Stockar, U.; Gustafsson, L.; Larsson, C.; Marison, I.; Tissot, P.; Gnaiger, E. Thermodynamic considerations in constructing energy balances for cellular growth. Biochimica et Biophysica Acta (BBA) Bioenergetics 1993, 1183, 221-240. doi:10.1016/0005-2728(93)90225-5.

5. von Stockar, U.; Liu, J.S. Does microbial life always feed on negative entropy? Thermodynamic analysis of microbial growth. Biochimica et Biophysica Acta (BBA) - Bioenergetics 1999, 1412, 191-211. doi:10.1016/S0005-2728(99)00065-1. 
6. von Stockar, U. Biothermodynamics of live cells: a tool for biotechnology and biochemical engineering. Journal of Non-Equilibrium Thermodynamics 2010, 35. doi:10.1515/jnetdy.2010.024.

7. von Stockar, U.; van der Wielen, L.A. Biothermodynamics: The role of thermodynamics in biochemical engineering; EPFL Press, 2013

Monod, J. Recherches sur la croissance des cultures bacteriennes 1942

Monod, J. The growth of bacterial cultures. Annual review of microbiology 1949, 3, 371-394.

Monod, J. La technique de culture continue: theorie et applications 1950.

Blackman, F. Optima and Limiting Factors. Ann. Bot. 1905, 19, 281.

2. Tessier, G. Croissance des populations bactériennes et quantité d'aliment disponible. Rev. Sci. Paris 1942, 80, 209.

13. Esener, A.A.; Roels, J.A.; Kossen, N.W.F. Theory and applications of unstructured growth models: Kinetic and energetic aspects. Biotechnology and Bioengineering 1983, 25, 2803-2841. doi:10.1002/bit.260251202.

14. Herbert, D.; Elsworth, R.; Telling, R.C. The Continuous Culture of Bacteria; a Theoretical and Experimental Study. Journal of General Microbiology 1956, 14, 601-622. doi:10.1099/00221287-14-3-601.

15. Herbert, D. VII Int. Congr. Microbiology. Recent progress in Microbiology 1958, p. 381.

16. Pirt, S. The maintenance energy of bacteria in growing cultures. Proceedings of the Royal Society of London. Series B. Biological Sciences 1965, 163, 224-231.

17. van Bodegom, P. Microbial Maintenance: A Critical Review on Its Quantification. Microbial Ecology 2007, 53, 513-523. doi:10.1007/s00248-006-9049-5.

18. Erickson, L.E.; Minkevich, I.G.; Eroshin, V.K. Utilization of mass-energy balance regularities in the analysis of continuous-culture data. Biotechnology and Bioengineering 1979, 21, 575-591. doi:10.1002/bit.260210405.

19. Roels, J.A. Application of macroscopic principles to microbial metabolism. Biotechnology and Bioengineering 1980, 22, 2457-2514. doi:10.1002/bit.260221202.

20. Roels, J.A. Simple model for the energetics of growth on substrates with different degrees of reduction. Biotechnology and Bioengineering 1980, 22, 33-53. doi:10.1002/bit.260220104.

21. Heijnen, J.J.; van Loosdrecht, M.C.M.; Tijhuis, L. A black box mathematical model to calculate auto- and heterotrophic biomass yields based on Gibbs energy dissipation. Biotechnology and Bioengineering 1992, 40,1139-1154. doi:10.1002/bit.260401003.

22. Heijnen, S.J. Thermodynamics of microbial growth and its implications for process design. Trends in Biotechnology 1994, 12, 483-492. doi:10.1016/0167-7799(94)90056-6.

23. Mayberry, W.R.; Prochazka, G.J.; Payne, W.J. Factors derived from studies of aerobic growth in minimal media. Journal of Bacteriology 1968, 96, 1424-1426.

24. Gunsalus, I.; Shuster, C. Energy-yielding metabolism in bacteria. In Metabolism; Elsevier, 1961; pp. 1-58.

25. Minkevich, I.G.; Eroshin, V.K. Productivity and heat generation of fermentation under oxygen limitation. Folia Microbiologica 1973, 18, 376-385. doi:10.1007/BF02875932.

26. Thornton, W. XV. The relation of oxygen to the heat of combustion of organic compounds. The London, Edinburgh, and Dublin Philosophical Magazine and Journal of Science 1917, 33, 196-203.

27. Patel, S.A.; Erickson, L.E. Estimation of heats of combustion of biomass from elemental analysis using available electron concepts. Biotechnology and Bioengineering 1981, 23, 2051-2067. doi:10.1002/bit.260230910. 28. Battley, E.H. Calculation of entropy change accompanying growth ofEscherichia coli K-12 on succinic acid. Biotechnology and Bioengineering 1993, 41, 422-428. doi:10.1002/bit.260410405.

29. Esener, A.A.; Veerman, T.; Roels, J.A.; Kossen, N.W.F. Modeling of bacterial growth; Formulation and evaluation of a structured model. Biotechnology and Bioengineering 1982, 24, 1749-1764. doi:10.1002/bit.260240803.

30. Scott, M.; Klumpp, S.; Mateescu, E.M.; Hwa, T. Emergence of robust growth laws from optimal regulation of ribosome synthesis. Molecular systems biology 2014, 10.

31. Weiße, A.Y.; Oyarzún, D.A.; Danos, V.; Swain, P.S. Mechanistic links between cellular trade-offs, gene expression, and growth. Proceedings of the National Academy of Sciences 2015, 112, E1038-E1047. doi:10.1073/pnas.1416533112.

32. Heijnen, J.J.; Van Dijken, J.P. In search of a thermodynamic description of biomass yields for the chemotrophic growth of microorganisms. Biotechnology and Bioengineering 1992, 39, 833-858. doi:10.1002/bit.260390806. 
33. Battley, E.H. Calculation of the heat of growth ofEscherichia coli K-12 on succinic acid. Biotechnology and Bioengineering 1991, 37, 334-343. doi:10.1002/bit.260370407.

34. Battley, E.H. An empirical method for estimating the entropy of formation and the absolute entropy of dried microbial biomass for use in studies on the thermodynamics of microbial growth. Thermochimica Acta 1999, 326, 7-15. doi:10.1016/S0040-6031(98)00584-X.

35. Bonetta, L. Whole-genome sequencing breaks the cost barrier. Cell 2010, 141, 917-919.

36. Kanehisa, M.; Goto, S. KEGG: kyoto encyclopedia of genes and genomes. Nucleic acids research 2000, 28, 27-30.

37. Karp, P.D.; Riley, M.; Paley, S.M.; Pellegrini-Toole, A. The metacyc database. Nucleic acids research 2002, 30, 59-61.

38. Price, N.D.; Reed, J.L.; Palsson, B.Ø. Genome-scale models of microbial cells: evaluating the consequences of constraints. Nature Reviews Microbiology 2004, 2, 886.

39. Kauffman, K.J.; Prakash, P.; Edwards, J.S. Advances in flux balance analysis. Current opinion in biotechnology 2003, 14, 491-496.

40. Edwards, J.S.; Palsson, B.O. Metabolic flux balance analysis and the in silico analysis of Escherichia coli K-12 gene deletions. BMC bioinformatics 2000, 1, 1 .

41. Oliveira, A.P.; Nielsen, J.; Förster, J. Modeling Lactococcus lactis using a genome-scale flux model. BMC microbiology 2005, 5, 39.

42. Oberhardt, M.A.; Puchałka, J.; Fryer, K.E.; Dos Santos, V.A.M.; Papin, J.A. Genome-scale metabolic network analysis of the opportunistic pathogen Pseudomonas aeruginosa PAO1. Journal of bacteriology 2008, 190, 2790-2803.

43. Shinfuku, Y.; Sorpitiporn, N.; Sono, M.; Furusawa, C.; Hirasawa, T.; Shimizu, H. Development and experimental verification of a genome-scale metabolic model for Corynebacterium glutamicum. Microbial cell factories 2009, 8, 43.

44. Molenaar, D.; Van Berlo, R.; De Ridder, D.; Teusink, B. Shifts in growth strategies reflect tradeoffs in cellular economics. Molecular systems biology 2009, 5 .

45. Kümmel, A.; Panke, S.; Heinemann, M. Systematic assignment of thermodynamic constraints in metabolic network models. BMC bioinformatics 2006, 7, 512.

46. Noor, E.; Bar-Even, A.; Flamholz, A.; Lubling, Y.; Davidi, D.; Milo, R. An integrated open framework for thermodynamics of reactions that combines accuracy and coverage. Bioinformatics 2012, 28, 2037-2044.

47. Mavrovouniotis, M.L. Estimation of standard Gibbs energy changes of biotransformations. Journal of Biological Chemistry 1991, 266, 14440-14445.

48. Ataman, M.; Hatzimanikatis, V. Heading in the right direction: thermodynamics-based network analysis and pathway engineering. Current opinion in biotechnology 2015, 36, 176-182.

49. Basan, M.; Hui, S.; Okano, H.; Zhang, Z.; Shen, Y.; Williamson, J.R.; Hwa, T. Overflow metabolism in Escherichia coli results from efficient proteome allocation. Nature 2015, 528, 99.

50. Beg, Q.K.; Vazquez, A.; Ernst, J.; de Menezes, M.A.; Bar-Joseph, Z.; Barabási, A.L.; Oltvai, Z.N. Intracellular crowding defines the mode and sequence of substrate uptake by Escherichia coli and constrains its metabolic activity. Proceedings of the National Academy of Sciences 2007, 104, 12663-12668.

51. Alzoubi, D.; Desouki, A.A.; Lercher, M.J. Flux balance analysis with or without molecular crowding fails to predict two thirds of experimentally observed epistasis in yeast. Scientific reports 2019, 9, 1-9.

52. Schaechter, M.; Maaløe, O.; Kjeldgaard, N.O. Dependency on medium and temperature of cell size and chemical composition during balanced growth of Salmonella typhimurium. Microbiology 1958, 19, 592-606.

53. Kjeldgaard, N.; Maaløe, O.; Schaechter, M. The transition between different physiological states during balanced growth of Salmonella typhimurium. Microbiology 1958, 19, 607-616.

54. Noor, E.; Bar-Even, A.; Flamholz, A.; Reznik, E.; Liebermeister, W.; Milo, R. Pathway thermodynamics highlights kinetic obstacles in central metabolism. PLoS computational biology 2014, 10, e1003483.

55. Niebel, B.; Leupold, S.; Heinemann, M. An upper limit on Gibbs energy dissipation governs cellular metabolism. Nature Metabolism 2019, 1, 125.

56. Evans, D.J.; Cohen, E.G.D.; Morriss, G.P. Probability of second law violations in shearing steady states. Phys. Rev. Lett. 1993, 71, 2401-2404.

57. Jarzynski, C. Nonequilibrium Equality for Free Energy Differences. Phys. Rev. Lett. 1997, 78, $2690-2693$. doi:10.1103/PhysRevLett.78.2690. 
538

539

540

541

542

543

58. Crooks, G.E. Entropy production fluctuation theorem and the nonequilibrium work relation for free energy differences. Phys. Rev. E 1999, 60, 2721-2726.

59. Evans, D.J.; Searles, D.J. The Fluctuation Theorem. Advances in Physics 2002, 51, 1529-1585. doi:10.1080/00018730210155133.

60. England, J.L. Statistical physics of self-replication. The Journal of Chemical Physics 2013, 139, 121923. doi:10.1063/1.4818538.

61. Piñero, J.; Sole, R. Nonequilibrium Entropic Bounds for Darwinian Replicators. Entropy 2017, 20. doi:10.3390/e20020098.

62. King, Z.A.; Lu, J.; Dräger, A.; Miller, P.; Federowicz, S.; Lerman, J.A.; Ebrahim, A.; Palsson, B.O.; Lewis,

N.E. BiGG Models: A platform for integrating, standardizing and sharing genome-scale models. Nucleic acids research 2015, 44, D515-D522.

63. Schill, N.A.; Liu, J.S.; Stockar, U.v. Thermodynamic analysis of growth of Methanobacterium thermoautotrophicum. Biotechnology and bioengineering 1999, 64, 74-81. 